\title{
Effect of valence holes on swift heavy ion track formation in $\mathrm{Al}_{2} \mathrm{O}_{3}$
}

P.N. Terekhin ${ }^{1}$, R.A. Rymzhanov ${ }^{2}$, S.A. Gorbunov ${ }^{3}$, N.A. Medvedev ${ }^{4}$, A.E. Volkov ${ }^{1,2,3}$

${ }^{1}$ National Research Centre 'Kurchatov Institute', Kurchatov Sq. 1, 123182

Moscow, Russia

${ }^{2}$ JINR, Joliot-Curie 6, 141980 Dubna, Moscow Region, Russia

${ }^{3}$ LPI of the Russian Academy of Sciences, Leninskij pr., 53,119991 Moscow, Russia

${ }^{4}$ Center for Free-Electron Laser Science at DESY, Notkestr. 85, 22607 Hamburg,

Germany

\begin{abstract}
This paper focuses on effects of electrons and valence holes on structure modifications in swift heavy ion (SHI) tracks in dielectrics. To investigate this problem a multiscale model is constructed which consists of (a) Monte Carlo modeling of a SHI penetration and secondary electron cascading; (b) molecular-kinetic approach for low-energy electrons spatial redistribution after finishing of ionization cascades; (c) molecular dynamics modeling of lattice excitation due to its coupling with the relaxing electron ensemble and subsequent atomic dynamics on picoseconds timescales. The model is applied to $167 \mathrm{MeV} \mathrm{Xe}$ ion irradiation of solid $\mathrm{Al}_{2} \mathrm{O}_{3}$. We found that lattice heating by excited electrons does not exceed $\sim 200 \mathrm{~K}$, which is not sufficient to form an ion track observed in experiments. Structure transformations appear in the numerical simulations only when the excess energy accumulated in valence holes is taken into account.
\end{abstract}

Keywords: swift heavy ion, dynamic structure factor, complex dielectric function, molecular dynamics, Monte-Carlo, electron-lattice coupling.

DOI:10.1016/j.nimb.2015.01.069 


\section{INTRODUCTION}

A swift heavy ion (SHI, $M>20$ a.m.u., $E>1 \mathrm{MeV} /$ nucl) loses a large part of its energy on excitation of the electron subsystem of a target in the nanometric vicinity of its trajectory (>95\%, $1-40 \mathrm{keV} / \mathrm{nm}$ along the projectile path) [1]. This energy loss occurs via target ionization resulting in generation of fast electrons. Propagation of these electrons from the SHI trajectory produces new ionizations forming next generations of electrons and holes. During the subsequent relaxation of the excited electron ensemble, a part of its excess energy is transferred to target atoms resulting in transient lattice excitation in an ion track.

Dissipation of the excess energy of delocalized electrons in SHI tracks occurs on shorter times than those of atomic vibrations and, thus, the characteristic time of the collective modes of a lattice [2]. Therefore, an adequate description of energy transfer from excited electrons to the lattice in a SHI track requires a more general approach than that based on electron-phonon coupling [3-6]. Such an approach must take into account possibilities of realization of various limit cases of dynamical response of lattice atoms during their interaction with the relaxing electron ensemble in a track.

The presented microscopic multiscale model describes the kinetics of excitation of a dielectric in a SHI track up to picoseconds after the projectile passage. It consists of three different approaches combined together [7,8]. At the first stage, up to tens of femtoseconds after the projectile passage, a Monte Carlo (MC) [9-11] model is applied to simulate initial excitations of the electron subsystem by an SHI as well as further kinetics of fast electrons appearing due to ionization. At longer times ( $>10 \mathrm{fs})$, spatial spreading of electrons is accounted for by means of the molecular-kinetic method. The energy and momentum exchange of excited electrons with the lattice is calculated tracing the atomic trajectories with the molecular dynamics (MD) method up to picoseconds after the SHI passage.

The model is applied to estimate lattice heating of $\mathrm{Al}_{2} \mathrm{O}_{3}$ in the track of $167 \mathrm{MeV}$ Xe ion. The simulations demonstrate that heating of $\mathrm{Al}_{2} \mathrm{O}_{3}$ due to coupling of its lattice to delocalized 
electrons is too low $(\sim 200 \mathrm{~K})$ to produce structure transformations detected in experiments [12]. Such structure transformations appear in the numerical simulations only when the lattice is additionally supplied with the excess energy accumulated in valence holes (simulated track has a diameter of $2 \mathrm{~nm}$ vs $1.3 \mathrm{~nm}$ track observed in the experiment [12]).

\section{MULTISCALE MODEL}

We apply an iterative scheme to estimate the spatial and temporal dependencies of the energy transferred into the lattice of $\mathrm{Al}_{2} \mathrm{O}_{3}$ from the excited electrons in a SHI track. First, the MC model is used to determine the initial radial distributions of the parameters characterizing an excited state of the ensemble of electrons: their concentration and the energy density. These initial distributions are used to restore the initial local equilibrium distribution functions of electrons at different distances from the axis of the track coinciding with the SHI trajectory. After finishing of ionization cascades, the molecular-kinetic method provides the changes of the concentration and the energy of low-energy electrons at different radii from the ion trajectory [8].

Using the dynamic-structure factor (DSF) formalism described below, the rates of the energy exchange of excited electrons with the lattice at different distances from the ion trajectory are calculated resulting also in decrease of the excess energy of the electron ensemble. The energy transferred from electrons to the lattice in different track regions is distributed among the different species of atoms by increasing their kinetic energy in accordance with their mass fractions.

MD tracing of atoms enables us to obtain the transient lattice DSF and, thus, realistic cross sections of the electron-lattice energy exchange in a relaxing SHI track. The same MD also models the lattice relaxation and spatial dissipation of the excess energy of the atoms after cooling of the electron ensemble [7,8]. The characteristic time of cooling of delocalized electrons in a SHI track due to their spatial spreading is shorter than the time of thermalization 
of the nonequilibrium lattice [13]. Therefore, we use the kinetic temperature of lattice atoms to estimate lattice heating during this exchange [14]:

$$
T_{k i n}(\mathbf{r}, t)=\frac{1}{P} \sum_{\alpha=1}^{P} T_{k i n, \alpha}(\mathbf{r}, t)=\frac{1}{P} \sum_{\alpha=1}^{P} \frac{M_{\alpha}}{3 k_{B}\left(Q_{\alpha}-1\right)} \sum_{n=1}^{Q_{\alpha}}\left(\mathbf{v}_{n}-\mathbf{v}_{\alpha}^{c}\right)^{2}
$$

Here $P$ is the number of different types of atoms of a target $\left(P=2\right.$ for $\left.\mathrm{Al}_{2} \mathrm{O}_{3}\right) ; k_{B}$ is the Boltzmann constant; $Q_{\alpha}$ are the numbers of atoms of each type $\alpha$ in a volume where the kinetic temperature is defined; $\mathbf{v}_{n}$ is the velocity of an $\alpha$-atom of number $n ; M_{\alpha}, \mathbf{v}_{\alpha}^{c}$ are the masses and velocities of the center of mass of $\alpha$-atoms in a cell which this $\alpha$-atom $n$ belongs to.

For estimation of the valence holes contribution to lattice excitation we use the simplest model of an instant energy deposition to the atomic system with subsequent lattice relaxation by means of MD. This crude procedure does not assume any particular physical mechanism governing such energy exchange, and is used merely for a 'zero-order' "upper" estimation of the effect of valence holes.

The details of different modules of the model are described in the next subsections. We would like to emphasize that the model does not require any knowledge in advance about the experimental track radius, it has a full predictive capability.

\section{II.1. Monte Carlo simulation of the initial kinetics of the electron ensemble in a swift heavy ion track}

The details of the numerics of the MC model used to describe the excitation of the electronic subsystem of a target are presented in Refs. [9-11]. At the first step, the model simulates a passage of an ion and its energy losses which result in creation of the first generation of excited electrons. The ion trajectory within our simulation slice of $10 \mathrm{~nm}$ is assumed to be a straight line along the Z-axis because of negligible energy transfer in a single 
ion-electron scattering event compared to the kinetic energy of a projectile due to the large difference in their masses.

The model is based on the cross sections of interaction of excited free electrons and an SHI with the electron and atomic subsystems of a target. A simple but efficient approach describing interaction of an incident projectile with a dynamically coupled system of particles is based on (a) the one-particle approximation for a projectile; and (b) the assumption of weak interaction of this projectile with the scattering system (the first Born approximation) [11]. In this case, the differential cross section is factored into the cross section describing scattering of a projectile on an isolated particle of the scattering ensemble, and the dynamic structure factor (DSF) describing a collective response of this ensemble to the transferred energy and momentum.

The fluctuation dissipation theorem links the DSF of the ensemble of charged particles with the complex dielectric function (CDF) of this ensemble (a target). This allows to use the experimental optical data for construction of the CDF-based cross sections used in the MC approach $[10,11]$. The CDF formalism allows us to reconstruct all the effects of the whole valence band of $\mathrm{Al}_{2} \mathrm{O}_{3}$ crystal, so we do not need to describe "partial" effects from valence electrons of the different kinds of atoms.

The mean free paths of electrons calculated within the CDF-formalism agree well with the NIST database as shown in Ref. [11] for high electron energies down to $\sim 50 \mathrm{eV}$. As it is known from the original work [15], the same formalism also yields a correct limit for low electron energies (below a few eV, due to a strong static screening). Potential problems can arise only in the intermediate energy range between a few $\mathrm{eV}$ and $\sim 50 \mathrm{eV}$. In this region, however, we use the same cross section assuming that possible deviations should not be too strong.

Propagation of electrons generated in an ion track is modeled even-by-event, accounting for the secondary impact ionizations leaving holes in deep and valence shells, and/or elastic 
scattering of electrons on lattice atoms. Within this manuscript the term 'elastic scattering' refers to the exchange of kinetic energy only, without ionization of the target atoms, while 'inelastic' refers to an ionization event. The transferred energy $\Delta E_{e}$ (and the corresponding zenith scattering angle $\theta$ ) of an ionized electron are calculated from the differential cross section in accordance with the energy and momentum conservations [11]. The azimuth angle of scattering $\varphi$ is chosen randomly between 0 and $2 \pi$. Auger decays of deep-shell holes are also modeled.

In this paper we do not take into account a possible effect of the potential of the positively charged track core because of its very rapid and nearly complete neutralization (on timescales $<1$ fs) [16]. The remaining long-living part, according to Schiwietz et al. [16], gives only a slight shift in the energy spectrum of delocalized electrons generated in a SHI track, thus having a weak effect on their spreading.

The MC procedure is iterated for $\sim 10^{4}$ times to obtain a trustworthy statistical averaging (error $<10^{-4}$ ). The averaged spatial distributions of the electrons concentration and their energy density are then extracted and used as the initial conditions for further modeling [17]. Figs. 1 and 2 present these initial radial distributions calculated in the layer of thickness of 10 $\mathrm{nm}$ along the ion path after an impact of $167 \mathrm{MeV} \mathrm{Xe}$ ion in $\mathrm{Al}_{2} \mathrm{O}_{3}$.

\section{II.2. Spatial spreading of delocalized electrons}

Equilibration between the electron and the lattice temperatures results mainly from spatial redistribution of excited electrons and their elastic interaction with lattice atoms after finishing of the ionization cascades (at times $\sim 10 \mathrm{fs}$ after the ion passage). Taking this into account, we use a simplified approach to describe the kinetics of the excited electron ensemble at these times.

Spatial gradients of the concentrations and average electron velocities govern the transport of electrons and their energy between different track regions. To describe this 
transport, the molecular-kinetic method is applied from the time when ballistic spatial spreading of excited electrons turns into the diffusive behavior [18], until the time when the electron temperature equilibrates with the atomic one. The details of this method can be found in Refs. [7,8].

\section{II.3. The electron-lattice energy exchange}

Within the one-electron approximation the energy transfer rate $Q=-d E / d t$ to the lattice due to elastic scattering of electrons is described by (see [2]):

$$
Q=\frac{\hbar^{4}}{2 \pi^{3} m_{e}^{2}} \int f_{\mathbf{k}_{\mathbf{i}}}\left(1-f_{\mathbf{k}_{\mathbf{f}}}\right) \omega \frac{k_{i}}{k_{f}} \frac{\partial^{2} \sigma}{\partial \Omega_{f} \partial E_{f}} d \mathbf{k}_{\mathbf{i}} d \mathbf{k}_{\mathbf{f}},
$$

here $m_{e}$ is the electron mass; $\hbar$ is the Plank constant; $f_{\mathbf{k}_{\mathbf{i}}}$ and $f_{\mathbf{k}_{\mathrm{f}}}$, are the equilibrium distribution functions of electrons, and $\mathbf{k}_{\mathbf{i}}$ and $\mathbf{k}_{\mathbf{f}}$ are, respectively, the initial and final wave vectors of the electron; $\hbar \omega$ is the change of the energy of the electron; $\Omega_{f}$ is the solid angle corresponding to the outgoing wave vector $\mathbf{k}_{\mathbf{f}}$, and $E_{f}=\hbar^{2} \mathbf{k}_{\mathbf{f}}^{2} / 2 m_{e}$ is an outgoing energy of the scattered electron.

As mentioned above, the cross section of scattering of excited electrons on the lattice is written via DSF $S(\mathbf{k}, \omega)[19]$ :

$$
\frac{\partial^{2} \sigma}{\partial \Omega_{f} \partial E_{f}}=|V(\mathbf{k})|^{2} \frac{m_{e}^{2}}{4 \pi^{2} \hbar^{5}} \frac{k_{f}}{k_{i}} S(\mathbf{k}, \omega)
$$

here $\mathbf{k}=\mathbf{k}_{\mathbf{f}}-\mathbf{k}_{\mathbf{i}}$ is the change of wave vector of the scattered electron; $V(\mathbf{k})$ is the spatial Fourier transform of the interaction potential between an electron and a single atom of a target. We use the Thomas-Fermi screened potential to describe this interaction:

$$
V(r)=\frac{Z_{a t} e}{4 \pi \varepsilon_{0} r} e^{-\frac{r}{L_{S C R}}}, \quad L_{S C R}^{-2}=\frac{e_{0}^{2}}{\varepsilon_{0}} \frac{\partial}{\partial \mu} \int \frac{d \mathbf{k}}{4 \pi^{3}} f_{k},
$$


where $Z_{a t}$ is a charge of a lattice ion; $\varepsilon_{0}$ is the vacuum permittivity; $L_{S C R}$ is a screening length $[20] ; \mu$ is a chemical potential of delocalized electrons.

The DSF is the Fourier transform of the spatial and temporal pair correlation function $G(\mathbf{r}, t)[19]$ describing coupled atomic dynamics in the lattice:

$$
S(\mathbf{k}, \omega)=\frac{N}{2 \pi} \int d t d \mathbf{r} \exp [i(\mathbf{k r}-\omega t)] G(\mathbf{r}, t)
$$

Here $\mathrm{N}$ is the number of scattering atoms.

The classical approximation of $G(\mathbf{r}, t)$ can be obtained from MD simulations [21]:

$$
G(\mathbf{r}, t)=\frac{1}{N}\left\langle\sum_{i, j=1}^{N} \delta\left(\mathbf{r}+\mathbf{R}_{i}(0)-\mathbf{R}_{j}(t)\right)\right\rangle
$$

where $\langle\ldots\rangle$ is the statistical averaging over the classical ensemble of the lattice atoms. The periodic boundary conditions and ergodic hypothesis are employed to restore $G(\mathbf{r}, t)$ obtaining statistically meaningful results [22]. It should be noted that when the temperature of a lattice is higher that the temperature of electrons Eqs. (2-6) describe the energy transfer from the lattice to electrons.

Scattering of an electron on a two-component atomic system is described by the paircorrelation function [23]:

$$
G(\mathbf{r}, t)=q_{1} q_{1} G_{1-1}(\mathbf{r}, t)+q_{1} q_{2} G_{1-2}(\mathbf{r}, t)+q_{2} q_{1} G_{2-1}(\mathbf{r}, t)+q_{2} q_{2} G_{2-2}(\mathbf{r}, t)
$$

here $q_{1}$ and $q_{2}$ are the model charges of the lattice ions (for $\mathrm{Al}_{2} \mathrm{O}_{3}, q_{1}=q_{\mathrm{Al}}=1.5237$, $q_{2}=q_{\mathrm{O}}=-1.0158$, i.e. the index " 1 " is referring to " $\mathrm{Al}$ " and the index " 2 " is referring to "O" [24]).

Since the DSF is asymmetrical function [25]: $S(\mathbf{k}, \omega)=\exp \left(-\frac{\hbar \omega}{T_{l}}\right) S(-\mathbf{k},-\omega)$, where $T_{l}$ is the lattice temperature, the following correction should be introduced in order to satisfy the 
necessary quantum-mechanical asymmetry of the DSF $S_{c l}(\mathbf{k}, \omega)$ obtained from the classical $G(\mathbf{r}, t)$, Eqs. (5) and (6) [25-28]:

$$
S(\mathbf{k}, \omega)=\frac{\frac{\hbar \omega}{T_{l}}}{1-\exp \left(-\frac{\hbar \omega}{T_{l}}\right)} S_{c l}(\mathbf{k}, \omega)
$$

The electron subsystem cools down quickly due to the diffusion of electrons from the track core $(<100 \mathrm{fs})$. This time is usually shorter than the characteristic time of atomic vibrations $[7,8]$, which is much shorter than a time needed for any significant changes in the structure or the dynamical properties of the material. Taking this into account, we neglect in this version of the model changes in the lattice DSF during this time and the same DSF is used in Eq. (2) at each step of the modeling before equilibration between the electron and lattice temperatures.

The applied MD uses the Verlet algorithm with the time-step of 1 fs to simulate atomic dynamics of a lattice. For $\mathrm{Al}_{2} \mathrm{O}_{3}$ we use the interatomic Vashishta's potential [24] in this MD.

Strong electronic excitations may transiently change the interatomic potential. Acting sufficiently long time (a few hundred femtoseconds) such potential changes can result in nonthermal melting effects [7,29-31]. This change of the potential can be also treated as an effect of valence holes. In this paper we estimate only one among possible effects of valence holes to track region excitation. A lack of non-thermal melting in a SHI tracks indicates that change in the interatomic potential, if any, likely to be of a minor importance in tracks, and therefore is ignored in this paper. Nevertheless, the effect of atomic ionizations on the interatomic potential requires a dedicated investigation beyond the scope of the present paper.

The applied MD model was already verified for DSF calculation in [7]. The same MD code is used to describe relaxation of the excited lattice after equilibration of the electron ensemble with the atomic subsystem. 


\section{RESULTS: HEATING OF THE $\mathrm{AL}_{2} \mathrm{O}_{3}$ CRYSTAL IN SHI TRACKS}

\section{VI.1. Heating by free electrons}

The calculated temporal dependencies of the radial distributions of the kinetic temperature of $\mathrm{Al}_{2} \mathrm{O}_{3}$ lattice in a track of $167 \mathrm{MeV}$ Xe ion are shown in Fig.3. It demonstrates that heating of the $\mathrm{Al}_{2} \mathrm{O}_{3}$ lattice in the track region by hot delocalized electrons does not exceed $200 \mathrm{~K}$. Such heating can not stimulate any structure transformations detected in experiments [12] assuming thermal mechanisms (the melting temperature is $2330 \mathrm{~K}$ [24]).

\section{VI.2. Lattice heating including an excess energy of valence holes}

As we have seen in Fig. 2, a significant amount of the excess energy is transiently stored in valence holes. In order to estimate the effect of valence holes on structure transformations, we use the simplest approximation of the instant energy deposition. We input their energy into the lattice, assuming these holes as immobile. That is, when additionally to the energy transferred to the lattice via its coupling with delocalized electrons, we increase the kinetic energies of lattice atoms in the simulation cell by instantaneous deposition of the energy accumulated in valence holes, assigning random directions to the additional momenta of atoms. This procedure does not assume any particular physical mechanism of the energy exchange between the holes and the lattice. It is only used here to illustrate the importance of the excess energy stored in holes for atomic system and stimulate more detailed studies of holes' contribution in SHI tracks.

For this estimation, we apply a classical molecular dynamics code LAMMPS [32] based on its standard interatomic potential in the Buckingham-type form as it was proposed in [33]. We must note that modeling with this potential also does not produce any phase transitions in a track, when only the electron energy transfer to the lattice is included. The supercell size used in these simulations is $9.4 \times 9.7 \times 7.7 \mathrm{~nm}^{3}$ with periodic boundary conditions. We used corundum structure of $\mathrm{Al}_{2} \mathrm{O}_{3}$ (space group R-3c with lattice parameters (4.762 4.762 12.9990 
90 120)), which consists of a distorted hexagonal close-packed oxide sublattice with $\mathrm{Al}^{3+}$ cations occupying two thirds of the available octahedral holes. The borders of the computational cell in the X-and Y-directions are cooled by the Berendsen thermostat. Track evolution is traced up to $50 \mathrm{ps,}$ after which the cell temperature dropped below $350 \mathrm{~K}$, so no structural changes are expected after this time.

The radial excess energy density profiles calculated within the MC model for Xe ion at $100 \mathrm{fs}$ are presented in Fig. 4. We use this calculated energy density of valence holes as input energy for the atoms in this MD simulation. The peak within $3 \AA$ radius was excluded due to the technical computational reasons. This does not affect the final results due to its very small radius. Size of this region is ten times smaller than the radius of initially exited region (see Fig. 5a) and contains less than $1 \%$ of atoms participating in structure transformations.

Experiments in [12] showed for low ion fluences individual tracks of the size $\sim 1.3 \mathrm{~nm}$ as a set of discontinuous regions with low density. Figs. 5a and $\mathrm{b}$ demonstrate relaxation of the primary excitation. The excess energy of valence holes produces structure transformation with the track radius of $\sim 2 \mathrm{~nm}$ of a similar structure and in qualitative agreement with the experiment [12].

The larger size of the calculated track radius vs the experimental one can be explained by the fact that we assumed very simple model of energy transfer from holes to the lattice. In particular, the valence holes were assumed to be immobile, as discussed above. Thus, all their energy is deposited into the lattice without spreading off the track core.

\section{CONCLUSIONS}

Combined microscopic model describing all the stages of excitation in a SHI track is presented. Simulations describe the kinetics of initial excitation of the electron ensemble of an $\mathrm{Al}_{2} \mathrm{O}_{3}$ target by a swift heavy projectile, the subsequent ionization cascades, spatial spreading and relaxation of excited electrons, Auger relaxation of deep holes, energy and momentum 
transfer from the excited electron subsystem into the lattice. The developed MD describes also subsequent lattice dynamics.

Application of the DSF-CDF formalisms gives abilities to take automatically into account possible realizations of various limit cases of the collective response of the electron subsystem and of the lattice on excitations at ultra-short spatial and temporal scales of the track kinetics.

Application of the developed model to $\mathrm{Al}_{2} \mathrm{O}_{3}$ after $167 \mathrm{MeV}$ Xe impact demonstrates that the lattice heating by the delocalized electrons $(\sim 200 \mathrm{~K})$ is not sufficient for creation of any structure transformations.

The energy transferred from the valence holes into the lattice was then taken into account in the simplest way in order to estimate the effect of this energy on the kinetics of structure transformations in a track. It is demonstrated that including the excess energy of valence holes can stimulate structure transformations with the sizes similar to those detected in the experiment [12]. These results demonstrate that the kinetics of the valence holes subsystem cannot be neglected when modeling SHI track formation in dielectrics. Hole-lattice interaction requires additional detailed study.

\section{ACKNOWLEDGEMENTS}

We thank Dr. V.A. Skuratov for helpful discussions of the experiments. Financial support from grant 13-02-1020 ofi-m of Russian Foundation for Basic Research - Russia is acknowledged by S.A.Gorbunov and A. E. Volkov.

\section{REFERENCES}

1. A. M. Miterev, Phys. Usp., 172 (2002) 1131.

2. A.E. Volkov, V.A. Borodin, Nucl. Instrum. Methods Phys. Res., Sect. B, 146 (1998) 137.

3. Z. Lin, L.V. Zhigilei, V. Celli, Physical Review B, 77 (2008) 075133. 
4. B. Rethfeld, A. Kaiser, M. Vicanek and G. Simon, Physical Review B, 65 (2002) 214303.

5. J. Vorberger, D.O. Gerike, International Symposium on High Power Laser Ablation, AIP Conf. Proc. 1464 (2012) 572.

6. M.W.C. Dharma-wardana, F. Perrot, Physical Review E, 58 (1998) 3705.

7. S.A. Gorbunov, P.N. Terekhin, N.A. Medvedev, A.E. Volkov, Nucl. Instrum. Methods Phys. Res., Sect. B, 315 (2013) 173.

8. S.A. Gorbunov, N.A. Medvedev, R. A. Rymzhanov, P.N. Terekhin, A.E. Volkov, Nucl. Instrum. Methods Phys. Res., Sect. B, 326 (2014) 163.

9. N.A. Medvedev, A.E. Volkov, K. Schwartz, C. Trautmann, Physical Review B, 87 (2013) 104103.

10. N.A. Medvedev, R.A. Rymzhanov, A.E. Volkov, Nucl. Instrum. Methods Phys. Res., Sect. B, 315 (2013) 85.

11. R.A. Rymzhanov, N.A. Medvedev, A.E. Volkov, Nucl. Instrum. Methods Phys. Res., Sect. B, 326 (2014) 238.

12. V.A. Skuratov, J. O’Connell, N.S. Kirilkin, J. Neethling, Nucl. Instrum. Methods Phys. Res., Sect. B, 326 (2014) 223.

13. V.P.Lipp, A.E.Volkov, M.V.Sorokin, B.Rethfeld, Nucl. Instrum. Methods Phys. Res., Sect. B, 269 (2011) 865.

14. J.G. Powles, G. Rickayzen, D.M. Heyes, Mol. Phys., 103 (10) (2005) 1361.

15. R.H. Ritchie, A. Howie, Philos. Mag., Vol. 36, No. 2 (1977) 463.

16. G. Schiwietz, K. Czerski, M. Roth, F. Staufenbiel, P.L. Grande, Nucl. Instrum. Methods Phys. Res., Sect. B, 226 (2004) 683.

17. M.C. Ridgway, T. Bierschenk, R. Giulian, B. Afra, M. D. Rodriguez, L.L. Araujo, A.P. Byrne, N. Kirby, O.H. Pakarinen, F. Djurabekova et al, Physical Review Letters, 110 (2013) 245502. 
18. O. Osmani, N. Medvedev, M. Schleberger, B. Rethfeld, Physical Review B, 84 (2011) 214105.

19. L. van Hove, Physical Review, 95 (1954) 249.

20. N. W. Ashcroft, N. D. Mermin, Solid state physics, ed. by Harcourt (Saunders College Publishing, USA, 1976), p. 523.

21. R. Aamodt, K.M. Case, M. Rosenbaum and P. F. Zweipel, Physical Review, 126 (1962) 1165.

22. M. Rieth, Nano-Engineering in Science and Technology, (World Scientific Publishing Co. Pte. Ltd., USA, 2003), p. 90.

23. A.B. Bhatia, D.E. Thornton, Physical Review B, 2 (1970) 3004.

24. P. Vashishta, R. K. Kalia, A. Nakano, J. P. Rino, J. Appl. Phys. 103 (2008) 083504.

25. J. P. Hansen, I. R. McDonald, Theory of Simple Liquids (Academic Press, London, 2006), p. 204.

26. T. Scopigno, U. Balucani, G. Ruocco, F. Sette, Physical Review E, 63 (2000) 011210.

27. D. J. González, L. E. González, J. M. López, M. J. Stott, J. Chem. Phys., 115 (2001) 2373.

28. R. Gerald, A. Geiger, Mol. Phys., 70 (1990) 465.

29. A. Rousse, C. Rischel, S. Fourmaux, I. Uschmann, S. Sebban, G. Grillon, Ph. Balcou, E. Förster, J.P. Geindre, P. Audebert et al., Nature, 410 (2001) 65.

30. V.V. Stegailov, Contrib. Plasma Phys., 50, No. 1 (2010) 31.

31. S. K. Sundaram, E. Mazur, Nature Mat., 1 (2002) 217.

32. http://lammps.sandia.gov

33. M. Matsui, Phys. Chem, Minerals, 23 (1996) 345. 


\section{Figure Captions}

FIG. 1. Radial distribution of the electron concentration in a track of $167 \mathrm{MeV} \mathrm{Xe}$ in $\mathrm{Al}_{2} \mathrm{O}_{3}, 10$ fs after the ion impact.

FIG. 2. Radial distributions of the energy density of electrons and valence holes in a track of $167 \mathrm{MeV} \mathrm{Xe}$ ion in $\mathrm{Al}_{2} \mathrm{O}_{3}, 10$ fs after the ion impact.

FIG. 3. Radial distributions of the kinetic temperatures of $\mathrm{Al}_{2} \mathrm{O}_{3}$ lattice in a track of $167 \mathrm{MeV} \mathrm{Xe}$ ion when only the electron-atom interaction was taken into account. The maximal value is achieved at $0.3 \mathrm{ps}$.

FIG. 4. Calculated radial distributions of the excess-energy density of different subsystems of $\mathrm{Al}_{2} \mathrm{O}_{3}$ at $100 \mathrm{fs}$ after impact of a $167 \mathrm{MeV}$ Xe ion. Black circles are the energy transferred to the optical phonons calculated in $\mathrm{MC}$, blue squares are the energy of valence holes, red triangles are the energy of the electrons, solid green line is the energy transferred into the lattice in MD calculations.

FIG. 5. Atomic snapshots of $\mathrm{Al}_{2} \mathrm{O}_{3}$ lattice in the nanometric vicinity of the trajectory of a $167 \mathrm{MeV} \mathrm{Xe}$ ion at $1 \mathrm{ps}$ (a) and $50 \mathrm{ps}$ (b) after the ion impact, when conversion of the excess energy of valence holes into lattice excitation is taken into account. Al atoms are blue dots, $\mathrm{O}$ atoms are red dots. The projection along c-axis ([0001] direction of the hexagonal cell, coinciding with the SHI trajectory) is shown. 


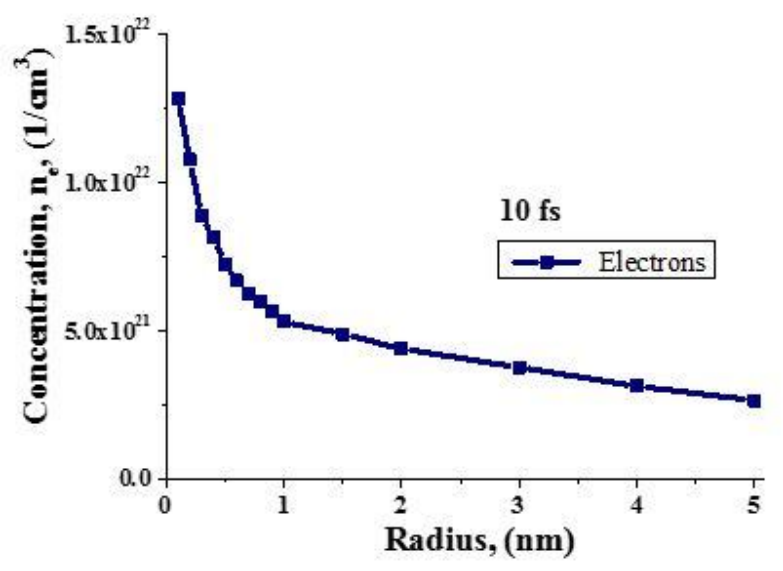

Figure 1 


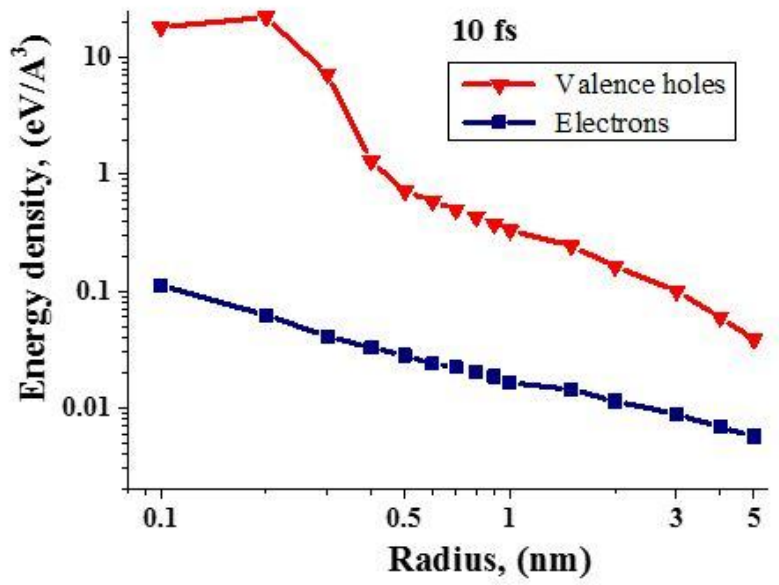

Figure 2 


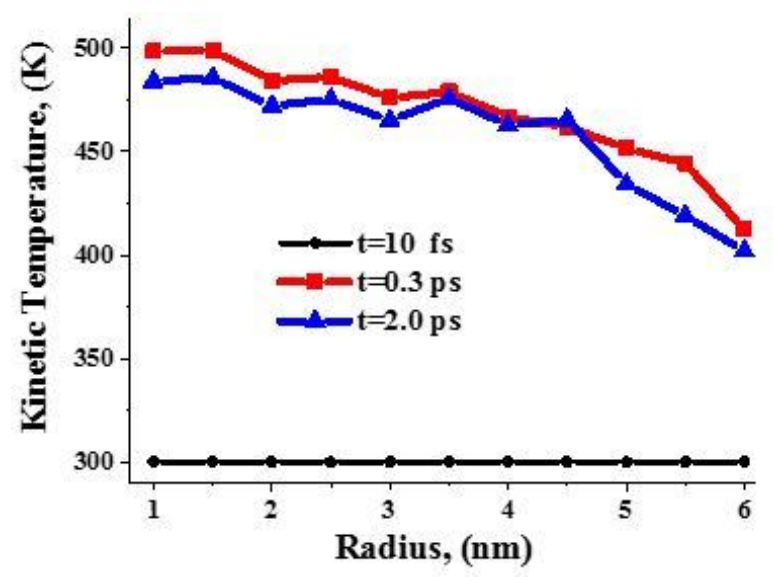

Figure 3 


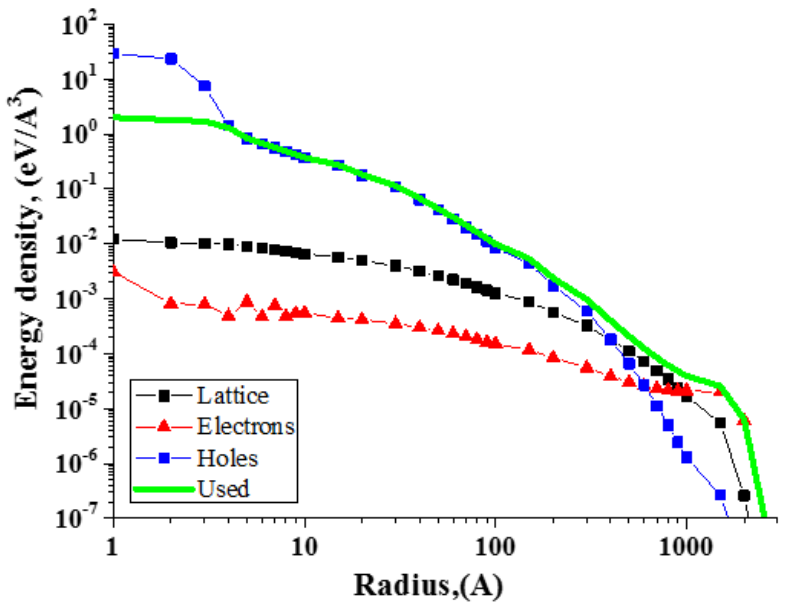

Figure 4 
a)

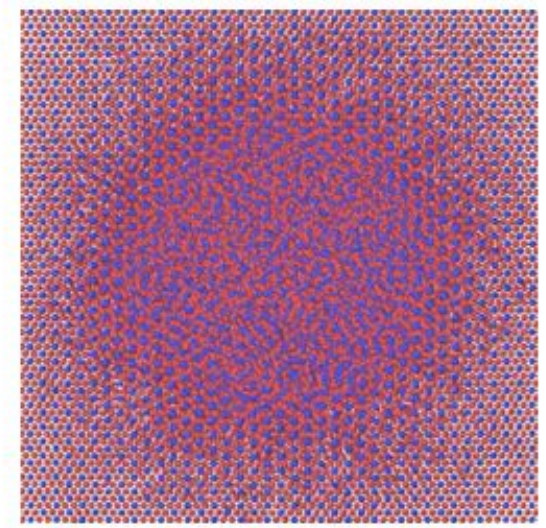

b)

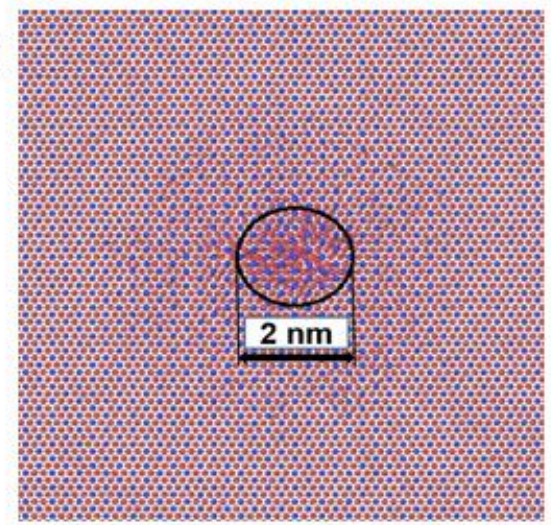

Figure 5 